\title{
Editorial, Volume 12, Issue 4, 2015
}

\author{
Rüdiger Schultz ${ }^{1}$
}

Published online: 26 October 2015

(C) Springer-Verlag Berlin Heidelberg 2015

This issue consists of five original papers of which the first three are forming a Featured Cluster

Stochastic Optimization in Electricity Production at CMS 2014 Lisbon.

The cluster has been initiated at the 11 th International Conference on Computational Management Science (CMS 2014) in Lisbon. The conference put emphasis on two topics of societal concern: Energy and Finance, partly mirrored in the title of the cluster. Apart from this focus, CMS 2014 has been a stimulating forum for theoreticians and practitioners from academia and industry to exchange knowledge, ideas, and results in a broad range, relevant to the theory and practice of computational methods in management science.

My sincere thanks go to the Co-Chairs of the Organizing Committee of CMS 2014, Raquel João Fonseca and João Telhada, both of the Operations Research Center of the University of Lisbon, for having arranged this conference so pleasantly, both scientifically and socially. I wish to extend my gratitude to Daniel Kuhn of the École Polytechnique Fédérale de Lausanne for his excellent service as Chair of the Program Committee. Last but not least, I want to thank my colleague Laureano Escudero of the Universidad Rey Juan Carlos, Mostoles/Madrid for his marvellous collaboration as Co-Editor of the present Featured Cluster.

The three papers in the cluster indicate the breadth of challenges in contemporary electricity production: starting from generation and transmission via consumption and offers into reserve electricity markets one finally arrives at dynamic programming based on approximate value functions and applied to hydroelectric production in multireservoir systems. A common thread of these papers is that there are decisions that,

Rüdiger Schultz

ruediger.schultz@uni-due.de

1 Faculty of Mathematics, University of Duisburg-Essen, 45127 Essen, Germany 
inevitably, must be taken in the presence of uncertainty expressable by probability distributions.

Motivated by limited stochastic modeling capabilities of current commercial software Francisco D. Muñoz and Jean-Paul Watson propose a scalable decomposition algorithm to solve stochastic transmission and generation investment planning problems with mixed discrete and continuous variables. They employ scenario reduction, to have a more tractable model, and scenario decomposition, with a version of the Progressive Hedging algorithm. Their numerical experiments with a 240-bus network representation of a real-life system lead to the conclusion that it is possible to obtain solutions with acceptable optimality gaps for practical applications.

Nigel Cleland, Golbon Zakeri, Geoff Pritchard, and Brend Young propose a model to optimize consumption level and associated reserve offer for a large consumer in a co-optimized electricity market. Their approach consists of three phases: potential energy and reserve prices under uncertainty are determined by simulation along with a full representation of the New Zealand electricity market dispatch model. Then the optimal reserve offer is found by dynamic programming, and the third phase repeats the first one with the optimal reserve offer intact. Initial experiments resulted in approaching a theoretical maximum profitability when using the model as an input to a site curtailment response strategy.

Luckny Zéphyr, Pascal Lang, and Bernard F. Lamond are dealing with a new approach to adaptive value function approximation in stochastic dynamic programming. Given convexity, their method works with simplicial partitions of the state space becoming finer and finer in the course of the algorithm. Refinement is guided by bounds on the value function. The method has been tested at reservoir management in hydro power generation. It led to the conclusion that the method is flexible in prescribing thresholds for relative imprecision. On the other hand, complexity of hyperrectangle decomposition a priorily limits the scope of the approach.

The two papers completing this issue somehow stand for the range of topics relevant to theory and practice of computational management science.

Joseph Andria, Giacomo di Tollo, and Raffaele Pesentini aim at finding optimal sizes for local tourism systems subject to geographical, economic, and demographical criteria. They formulate optimization problems, solve them by metaheuristics, and compare their results with the application of standard clustering techniques and exact solvers.

Composite vector variational-like inequalities and their relations to vector optimization problems are established by Anurag Jayswal, Shipra Singh, and Sarita Choudhury. Moreover, the authors derive sufficient conditions for the existence of solutions to composite vector variational-like inequalities and point to applications involving Nash equilibria.

This Editorial shall not be concluded without thanking the referees for their invaluable support. 